\title{
The Capillary Network: A Link between IVIM and Classical Perfusion
}

\author{
DENIS LE BIHAN* $\dagger$ AND ROBERT TURNER $\ddagger$ \\ * Diagnostic Radiology Department, The Warren G. Magnuson Clinical Center, and $\$$ National Heart, \\ Lung and Blood Institute, National Institutes of Health, Bethesda, Maryland 20892
}

Received April 30, 1991; revised September 17, 1991; accepted October 3, 1991

\begin{abstract}
MR measurements based on motion encoding gradients, such as intravoxel incoherent motion imaging, could provide, in principle, information on flowing blood volume and blood velocity. This note shows that, in addition, the knowledge of the capillary network organization may provide a link between these measurements and those obtained by conventional and MR perfusion techniques based on tracer uptake by tissues. (1) 1992 Academic Press, Inc.
\end{abstract}

\section{INTRODUCTION}

Recently, Henkelman ( 1 ) addressed a significant and fundamental issue: are phasesensitive MR techniques, such as intravoxel incoherent motion (IVIM) imaging (2), capable of measuring "classical" perfusion? What is under debate is not whether perfusion imaging using IVIM MR imaging is technically viable, but rather the concept of perfusion measurement with those methods. In his paper, Henkelman equates classical perfusion to tracer terminal deposition or uptake of tracers in tissues, which is measured with conventional methods, and concludes that phase-sensitive NMR imaging methods, in principle, do not measure classical perfusion, but "blood volume transit," unless assumptions are made regarding vascular structure and function. This problem is of importance, since it challenges the feasibility of perfusion measurement by directly monitoring the circulation of blood in tissues. A similar question was already addressed in the past (3), questioning the possibility of measuring perfusion by using pure intravascular tracers, such as with iodine-enhanced X-ray-computed tomography (4).

We have found the pertinent arguments of Henkelman of considerable interest in highlighting the application of the recently suggested NMR approaches to perfusion, and these have encouraged us to clarify the link that must exist between measurements obtained with tracer diffusion methods and the IVIM approach, based on our knowledge of the capillary network organization. The purpose of this note is to provide this link and to explain how IVIM imaging might give access to classical perfusion, for instance in the brain. The physiological basis for this link is epitomized by the classical

$\uparrow$ To whom reprint requests should be addressed at Diagnostic Radiology Department, Bldg. 10, Rm. IC660, The Warren G. Magnuson Clinical Center, National Institutes of Health, Bethesda, MD 20892. Fax (301)496-9933. 
central volume theorem, which serves as a basis for intravascular tracer methods and could be applied to other phase-sensitive MR techniques. Therefore, although the technical feasibility of perfusion measurements using IVIM imaging remains under debate, we felt that this conceptual discussion will be useful to other researchers working on perfusion MR imaging.

\section{THE CONCEPT OF PERFUSION}

Blood perfusion of tissues assures the delivery of oxygen and nutrients to cells. To achieve this goal, fresh, oxygenated "arterial" blood is delivered to the tissue through the capillary network, which is optimized for blood-tissue exchanges. Oxygen and nutrients are transported, passively or actively, in the tissue through the capillary wall, to be used by cells. Tissue metabolism results in waste products which are eliminated through the capillary network to constitute deoxygenated "venous" blood. These processes depend on many parameters, such as blood pressure, blood velocity, capillary network density and geometry, capillary wall permeability, nutrient or oxygen diffusion, and extraction rates, which can be grouped in three categories: microvascular anatomy and histology, blood microcirculation, and blood-tissue exchanges. For the physiologist, perfusion refers to the circulation of blood. However, in the literature the term "perfusion" has been sometimes used ambiguously in relation to any of these elementary categories. At the extreme, in the loosest sense of the term, perfusion has often been used by pathologists (and radiologists) simply to denote the degree (density) of normal or abnormal microvasculature in a tissue, as seen, for instance by conventional catheter angiography.

Conventional perfusion measurement techniques based on the uptake or washout of radionuclide tracers have unequivocally led to the identification of perfusion with "blood flow" in the tissue (cerebral blood flow [CBF] in the brain) measured in milliliters of blood $/ \mathrm{min} / 100 \mathrm{~g}$ of tissue. Blood-tissue exchange is a different process, which depends on the molecular species under study and which must not be confused with tissue perfusion. Let us consider a radiator made of coils through which hot water circulates. Obviously, the purpose of this radiator is to radiate heat into the room in which it lies. However, we should separate two distinct functional phenomena. First, hot water must enter, circulate, and leave the radiator. The flow of water circulating in the radiator is a well-defined quantity which can be measured. Second, the heat must be transmitted to the room, by diffusion, convection, or radiation, which may also be measured. Although the heat transfer depends on the water circulation in the radiator, water circulation and heat transfer are different entities which are described using different units. One can imagine, however, that one could possibly evaluate the water flow in the radiator from the temperature of the room, assuming some hypotheses. Similarly, one should distinguish between diffusible tracer perfusion measurement methods, which evaluate perfusion through tissue tracer concentrations, to pure intravascular tracer techniques, which directly evaluate the water pipe flow. In both cases, however, only blood flow is the bottom line quantity which one desires ultimately measure.

We shall now discuss in more detail what is actually measured by conventional tracer methods, and how NMR methods, especially IVIM imaging, may be compared to them. 


\section{PERFUSION, AS SEEN WITH CONVENTIONAL METHODS}

\section{Freely Diffusible Tracers}

Blood flow measurements are based on the application of the well-known Fick principle (5), which simply expresses mass conservation. In a differential form, the quantity of any substance (tracer) taken up per unit of time by an organ from the blood which perfuses it is equal to the amount of the substance carried to the organ by the arterial inflow less the amount removed by the drainage during the same unit of time. Blood flow (BF) is physically described in terms of the absolute volume of blood delivered per minute to the whole organ $(\mathrm{ml} / \mathrm{min})$. To normalize blood flow and provide an "intensive" unit independent of the organ size (a bigger organ requires more blood flow), it is usual to report blood flow by unit of organ mass, $\mathbf{M}$, assuming all substance is delivered and used uniformly by the organ. The quantity $(\mathrm{BF} / \mathrm{M})$ is a normalized volumetric flow rate $(\mathrm{ml} / \mathrm{min} / 100 \mathrm{~g})$ and corresponds well to the physiologist blood flow or perfusion (CBF in the brain).

Another important and usual assumption is that the venous concentration of the tracer is proportional to the tissue concentration at all times, assuming venous blood in the tissue is in instantaneous or fast equilibrium with the tissue itself $(6)$. The proportionality coefficient is called the partition coefficient. Blood flow (BF/M) can thus be determined from the respective arterial blood and tissue concentration time course, knowing the partition coefficient. One may monitor the progressive saturation of the tissue (wash-in methods) by the tracer, or the desaturation of the tissue (washout methods), or wait for a steady state to appear (7). This principle has fueled many methods based on different tracers $(8-11)$ or imaging modalities, such as SPECT (15, $16)$ or PET (17-21). In practice, one has to deal with additional refinements to take into account unsteady inflow concentrations, tracer recirculation or metabolism by other organs, partitioning of tracers in more than two compartments, diffusion rates ... $(12-14,22)$.

This type of blood flow measurement has the virtue of excluding the blood that could have only transited without exchange with the organ, since it is derived from the tracer concentration within the tissue. However, the bottom line parameter, here, is blood flow and not the exchange of the tracer with the tissue, since most often these tracers are not biological nutrients, so that their diffusion and compartmentalization in the tissue does not reflect physiological blood-tissue exchanges. The diffusion of the tracer in the tissue is just a technical artifice to measure blood flow.

\section{Nondiffusible Tracers}

Nondiffusible tracers have also been proposed to provide estimates of perfusion (4). Since by definition such tracers do not enter the tissue, the principle of mass conservation must be reformulated according to the central volume theorem (23), which states that absolute blood flow (BF) is

$$
\mathrm{BF}=\mathrm{Vd} / \mathrm{MTT} \text {, }
$$

where Vd is the volume of distribution of the tracer and MTT is its mean transit time. Vd has nothing to do with the organ volume and can be plasma, total blood, extracellular water, and so on. If the tracer is purely intravascular, $\mathrm{Vd}$ represents the plasma 
volume (taking into account the hematocrit) and can be calculated from the integral over time of the tracer concentration in the tissue vascular compartment $(13,14)$. MTT represents the average time required by a molecule or particle of the tracer to pass through the tissue and is usually difficult to evaluate, since the tissue tracer concentration time course is the product of convolution of the actual tissue residue function and the arterial input function $(13,14)$. It results that intravascular agents are most often used only to determine blood volume, which is not perfusion or blood flow (3).

In the ideal case, BF is now defined by the absolute volumic flow rate of the tracer passing through the tissue of interest (in $\mathrm{ml} / \mathrm{min}$ ). One may want again, for comparison purposes, to normalize $\mathrm{BF}$ to $\mathrm{M}$, so that $\mathrm{BF} / \mathrm{M}$ is also measured in $\mathrm{ml} / \mathrm{min} / 100 \mathrm{~g}$ of tissue. However, its significance may sometimes differ from that measured with diffusible tracers. One may easily imagine, depending on the tissue involved, that varying quantities of blood may simply be shunted through anatomoses from the arterial to the venous circulation, without being exchanged with the tissue, or simply, the measurement may also include a nonnegligible contribution from feeder or draining vessels. This "unused" blood will be included in the measurement, although it does not actually perfuse the tissue, in the physiological sense.

When applying these concepts to imaging, we must now refer to the apparent voxel scaling difficulties reported by Henkelman (1). A voxel of linear dimension $a$ has a volume and mass scaling as $a^{3}$. The absolute blood flow BF ( $\left.\mathrm{ml} / \mathrm{min}\right)$ passing through the voxel is $v s$, where $v$ is blood velocity and $s$ is the total capillary cross section on the voxel entry side. If the ratio $s / a^{2}$ is constant, the normalized volumetric flow rate or blood flow (in $\mathrm{ml} / \mathrm{min} / 100 \mathrm{~g}$ ) measured in the voxel, which is proportional to $v s /$ $a^{3}$, scales as $v / a$, and apparently depends on the voxel size. A logical normalization quantity would then be the section of entry of flow $\left(a^{2}\right)$, resulting in the cerebral blood fluence $\left(\mathrm{ml} / \mathrm{min} / \mathrm{cm}^{2}\right)$ of Henkelman.

However, our point of view differs from that of Henkelman when we consider the physiology which tells us that capillaries do not lie in the tissue just to assure blood circulation, but to permit exchanges of nutrients and oxygen to tissue. In other words, they constitute a functional unity such that (a) all blood entering a voxel will leave the voxel (no blood accumulation), and (b) capillaries are organized in parallel and not in series. The first statement means that monitoring the movement of blood itself in a voxel is equivalent to monitoring the transit of a pure intravascular tracer in this voxel. The second statement signifies that a perfusion measurement derived from an intravascular tracer is, indeed, an intensive quantity, as expected for blood flow, i.e., the measurement does not depend on the size of the voxel, and the measurement in each voxel can be integrated in a meaningful way to show total blood flow into the whole organ.

To simplify, let us assume that the parallel capillary "pipes" of a single voxel are fed by a common larger vessel (precapillary artery) of section $s$. To supply a voxel with a quantity $Q$ of nutrient per time unit, this larger vessel carries blood which flows with the velocity $v$. If the volume of the voxel is doubled, a quantity $2 Q$ must be delivered. This means that either a larger vessel (or two vessels) or a higher velocity is now necessary, so that $v$ s indeed scales as $a^{3}$, justifying a volume normalization. To quote the example given by Henkelman, this situation means that the drivers of 
United Parcel Service are efficient, so that statistics derived from the driver circulation pattern in a limited area do actually reflect the delivery of parcels to customers.

\section{PERFUSION, AS SEEN BY MRI}

Several methods have been proposed to image or measure perfusion by NMR. Again, the term perfusion has been used rather loosely, and we must give a tentative classification.

\section{Exogenous Tracer Methods}

Some methods are directly equivalent to the conventional approaches. They use external tracers which are administered to the animal or the patient, and are thus somewhat "invasive." NMR is only used as a detector. The approaches based on nonproton nuclei, such as ${ }^{19} \mathrm{~F}$-compounds $(24-26), \mathrm{D}_{2} \mathrm{O}(27)$, are in many instances comparable to the diffusible tracer methods and should give perfusion measurements with the limits and assumptions inherent to these methods. Other NMR techniques, based on proton NMR and paramagnetic/susceptibility contrast agents, such as GdDTPA, have been used in the brain where those tracers are considered to remain in the blood stream and do not diffuse in the tissue due to the presence of the bloodbrain barrier $(28,29)$. Therefore, they may be considered equivalent to the nondiffusible tracer approach and share their advantages and pitfalls. In particular they may be used to determine blood volume, but the evaluation of perfusion, i.e., blood flow remains difficult while the mean transit time is not determined accurately (3). Furthermore, one should remain cautious regarding the inclusion of "unused" blood in the measured blood flow. In particular, it is known from PET studies that a significant part of the circulating blood may come from other vessels than capillaries, such as small arteries and postcapillary veins. Most models assume, for simplicity, that these vessels are "invisible," which could lead to an overestimation of perfusion. Also, considering that water exchange between blood and tissue may not be negligible on a time scale of several seconds (30), these techniques may also share, in some circumstances, some features of the diffusible tracer approach $(3 I)$.

\section{Specific MR Methods-The IVIM Approach}

On the other hand, several methods have been suggested which do not use any external tracers, but exploit the natural sensitivity of the NMR signal to spin motion, using either time-of-flight $(32)$ or phase effects $(2,33)$. Flowing blood thus acts as an internal marker, so that these methods are totally noninvasive. Assuming water exchanges between blood and tissue are negligible at the measurement time scale (typically a few tens of milliseconds) which is reasonable in the brain $(30,34)$, these methods have some common ground with those using pure intravascular tracers. However, they also offer original, new insights to the problem of perfusion, which were not addressed by conventional methods. As an example, IVIM imaging is supposed to provide, in principle, information on blood microcirculation from two parameters (2), the "perfusion factor," $f$, and the "pseudo-diffusion coefficient," $D^{*}$. The contribution from larger (feeding or draining) vessels, in which blood velocity is higher 
is more or less spoiled by the presence of the magnetic-pulsed gradients. The quantity $f$ is the fractional volume (\%) of capillary blood flowing in each voxel, or more exactly the ratio of the volume of NMR-visible water flowing in the capillary compartment (plasma and blood cell content), Vd, to the total voxel volume of NMR-visible water $V_{\mathrm{H}_{2} \mathrm{O}}$, and can be converted into milliliters of capillary blood (corrected for its NMRvisible water content) per $100 \mathrm{~g}$ of tissue ( $\mathrm{CBV}$ ), knowing the tissue NMR-visible water content fraction $\left(f_{\mathrm{w}}=V_{\mathrm{H}_{2} \mathrm{O}} / V\right)$ and assuming tissue density is unity:

$$
f=\mathrm{Vd} / V_{\mathrm{H}_{2} \mathrm{O}}=\mathrm{Vd} / f_{\mathrm{w}} V=\mathrm{CBV} / f_{\mathrm{w}} .
$$

The pseudo-diffusion coefficient $D^{*}$ comes from the model in which, due to the quasi-random geometrical disposition of the capillary network (at least in the brain), blood microcirculation is regarded as a "diffusion" process, at a macroscopic scale. Therefore, one has for $D^{*}$

$$
D^{*}=\langle l\rangle\langle v\rangle / 6,
$$

where $\langle v\rangle$ is the average blood velocity and $\langle l\rangle$ is the mean capillary segment length (2). These two parameters may provide useful data for microcirculation physiology. For instance, when blood flow increases in a particular region of the brain as a response to a sensory stimulus, the physiologist is interested to know whether the mechanism is capillary recruitment (increase in $f$ ) or increase in blood velocity (increase in $D^{*}$ ). It is difficult to answer this question with conventional methods employing diffusible tracers. By monitoring separately $f$ and $D^{*}$, the IVIM approach has, in principle, the potential to solve the issue. On the other hand, if one wishes, IVIM results could be given in blood flow units (CBF). Let us combine the central volume theorem (Eq. [1]) with Eq. [2]:

$$
\mathrm{CBF}=\mathrm{CBV} / \mathrm{MTT}=f_{\mathrm{w}} f / \mathrm{MTT} .
$$

If $L$ is the total capillary length, one has, according to the definition of the mean transit time in the capillary network, and using Eq. [3],

$$
\operatorname{MTT}=L /\langle v\rangle=L\langle l\rangle / 6 D^{*} .
$$

Combining Eq. [4] and [5], one obtains

$$
\mathrm{CBF}=\left(6 f_{\mathrm{w}} / L\langle l\rangle\right) f D^{*} .
$$

An expression similar to Eq. [6] has been derived using a microscopic approach (35). Equation [6] shows that the link between classical perfusion and IVIM resides in the capillary geometry, and more especially in two lengths; the capillary segment length and the total capillary length. The quantity in parentheses, which depends on these two lengths, is thus constant for a given tissue, so that relative perfusion or blood flow can be estimated from the product $f D^{*}$. Furthermore, the mean transit time could also be evaluated (at least in a relative manner) from $D^{*}$ (Eq. [5]) without the need for any arterial "input function" $(13,14)$ and will not include the transit time of blood in the large feeding vessels. This is a legitimate hypothesis when one considers that blood velocity in feeder or draining vessels is somewhat larger, resulting in signal cancellation when the gradient pulses of the IVIM sequence are applied (2). In fact, 
a more sophisticated model should consider that $f$ and $D^{*}$ are also distributed and represent a continuum of values.

Relative measurements are useful, especially in functional studies when one compares symmetrical areas of an organ or when one monitors the perfusion response to physiological challenges. Absolute measurements would be possible in specific cases, only when a comprehensive knowledge of the microvascular anatomy is made available, as pointed out by Henkelman. For instance, in the cat brain cortex, one has $L=2$ $\mathrm{mm}$, and $\langle l\rangle=108 \mu \mathrm{m}(36)$. Typical IVIM data are $f=6.1+/-0.4 \%, D^{*}=11+/$ $-210^{-9} \mathrm{~m}^{2} / \mathrm{s}$ in the cat brain cortex (37). Taking $f_{\mathrm{w}}=0.78(38)$, one obtains from Eq. [2] CBV $=4.8+/-0.3 \mathrm{ml} / 100 \mathrm{~g}$, from Eq. [5] MTT $=3.4+/-0.6 \mathrm{~s}$ and from Eq. [6] $\mathrm{CBF}=87+/-22 \mathrm{ml} / \mathrm{min} / 100 \mathrm{~g}$, which is in excellent agreement with literature data (39), given the approximations made on the anatomical and IVIM measurements.

\section{CONCLUSION}

This high degree of consistency is very encouraging, although considerably more work remains to be done. Depending on the organ and the measurement time, bloodtissue water exchanges may also play some role $(30,34,40)$, so that the IVIM approach could also share some of the features of the diffusible tracer techniques. Indeed, the first issue to consider is technical, since it remains to be unequivocally demonstrated that perfusion can be reliably separated from the massive contribution of bulk tissue water. Some encouraging results have been obtained in tissue with high perfusion, such as kidney (4l), while recent brain studies have highlighted the extreme difficulty of the technique $(42,43)$. A possible means would be to artificially decrease the contribution of bulk tissue water, as has been recently proposed (44). This conceptual link between IVIM parameters and classical perfusion may help to compare IVIM imaging with established techniques. Similar links should be applicable to other perfusion MR techniques. These NMR-specific techniques have the potential not only to give perfusion measurements in the classical sense, but also to provide information on perfusion which is not always available with conventional tracer based methods, justifying the continuation of efforts in this exciting and promising area of research.

\section{ACKNOWLEDGMENT}

This note has been made possible thanks to discussions and arguments with many colleagues, NMR researchers, and physiologists, in particular, Drs. C. T. W. Moonen and J. Pekar. Dr S. S. Kety is gratefully acknowledged for his invaluable comments on the concept of perfusion.

\section{REFERENCES}

1. R. M. Henkelman, Magn. Reson. Med. 16, 470 (1990).

2. D. Le Bihan, E. Breton, D. Lallemand, M. L. Aubin, J. Vignaud, and M. Laval-Jeantet, Radiology, 168, 497 (1988).

3. N. A. LASSEN, J. Cereb. Blood Flow Metab. 4, 633 (1984).

4. L. AXEL, Radiology 137, 679 (1980).

5. A. FicK, Verhandl. d. phys.-med. Ges. zu Wurzburg. 2:xvi, 1870. (quoted in its entirety by H. E. Hoff and H. J. Scott, N. Engl. J. Med. 239, 122, 1948).

6. N. ZuNTZ, Fortschr. d. Med. 15, 632 (1897).

7. S. S. KETY, Am. Heart J. 38, 312 (1949). 
8. S. S. KetY ANd C. F. SCHMidt, Am. J. Physiol. 143, 53 (1945).

9. N. A. LASSEN, I. F. LiNDBJERG, AND O. MUNCK, Lancet, 686 (1964).

10. D. H. Ingvar and N. A. Lassen, Acta Physiol. Scand. 54, 325 (1962).

11. W. D. Obrist, H. K. Thompson, H. S. WANG, AND W. E. Wilkinson, Stroke 6, 245 (1974).

I2. S. S. KETY, Pharm. Rev. (1951).

13. K. L. ZIERLER, Circ. Res. 10, 393 (1962).

14. K. L. ZiERLER, Circ. Res. 16, 309 (1965).

15. E. M. Stokely, E. Sveinsdottir, N. A. LAssen, ANd P. J. Rommer, Comput. Assist. Tomogr. 4, 230 (1980).

16. D. Gur, W. F. Good, S. K. Wolfson, H. Yonas, and L. Shabason, Science 215, 1267 (1982).

17. M. M. Ter-Pogossian, J. O. Eichling, D. O. Davis, M. J. Welch, AND J. M. MEtzger, Radiology 93, 31 (1969).

18. P. HerscovitCh, J. Markham, And M. E. Raichle, J. Nucl. Med. 24, 782 (1983).

19. J. C. Baron, D. Comar, F. Soussaline, A. Todd-Pokropek, M. G. Bousser, P. Castaigne, and C. Kellershohn, Acta Neurol. Scand. 60 (Suppl 72), 194 (1979).

20. R. S. J. Frackowiak, G. L. Lenzi, T. Jones, and J. D. Heather, J. Comput. Assist. Tomogr. 4, 727-736, 1980.

21. T. Jones, D. A. Chessler, And M. M. Ter-Pogossian, Br. J. Radiol. 49, 339 (1976).

22. H. K. Thompson, C. F. Starmer, R. E. Whalen, and H. D. McIntosh, Circ. Res. 14, 502 (1964).

23. G. N. Steward, J. Physiol. 15, 1 (1894).

24. N. M. Bolas, A. J. Petros, D. Bergel, and G. K. Radda, "Book of Abstracts: Society of Magnetic Resonance in Medicine, 1985," p. 315.

25. D. Barranco, L. N. Sutton, S. Florin, J. Greenderg, T. Sinnwell, L. Ligeti, and A. C. MCLaughlin, J. Cereb. Blood Flow Metab. 9, 88, 6 (1989).

26. J. R. Ewing, C. A. Branch, J. A. Helpern, M. B. SChmith, S. M. Butt, and K. M. A. Welch, Stroke 20, 259 (1989).

27. J. J. H. Ackerman, C. S. Ewy, N. N. Becker, and R. A. Shalwitz, Proc. Natl. Acad. Sci. USA 84, 4099 (1987).

28. J. W. Belliveau, B. R. Rosen, H. L. Kantor, et al. Magn. Reson. Med. 14, 538 (1990).

29. J. W. Belliveau, B. R. Rosen, H. L. Kantor, el al. Magn. Reson. Med. 14, 538 ( 1990 ).

30. J. O. Eichling, M. E. Raichle, R. L. Grubb, And M. M. Ter-Pogossian, Circ. Res. 35, 358 ( 1974 ).

31. G. Sobering, C. T. W. MoOnen, AND J. A. FranK, "Book of Abstracts: Society of Magnetic Resonance in Medicine, 1990," p. 53.

32. J. A. Detre, J. S. Leigh, D. S. Williams, AND A. P. KoretSky, "Book of Abstracts: Society of Magnetic Resonance in Medicine, 1990," p. 1289.

33. I. R. Young, A. S. Hall, D. J. BRyant, et al. J. Comput. Assist. Tomogr. 12(5), 721 (1988).

34. P. A. Fraser and A. D. Dallas, J. Physiol. 423, 343 (1990).

35. R. Turner and P. Kellog, Prog. NMR Spectrosc. 23, 93 (1991).

36. G. PAWLIK, A. RACKL, AND R. J. BING, Brain Res. 208, 35 (1981).

37. D. Le Bihan, C. T. W. Moonen, P. C. M. van Zijl, J. Pek ar, and D. DesPres, J. Comput. Assist. Tomogr. 15, 19 (1991).

38. P. P. Fatouros, A. Marmarou, S. J. Cothran, K. A. Kraft, D. J. Deyo, and F. J. Laine, "Book of Abstracts: Society of Magnetic Resonance in Medicine, 1991," p. 47.

39. R. L. Grubb, M. E. Raichle, J. O. Eichling, and M. M. Ter-Pogossian, Stroke 5, 630 ( 1974 ).

40. G. T. Gullberg, X. MA, D. L. Parker, ANd D. N. Ghosh, Roy, Magn. Reson. Imaging 9, 39 ( 1991 ).

41. T. A. Powers, C. H. Lorentz, G. E. Holburn, And R. R. Price, Radiology 178, 543 (1991).

42. R. C. MCKinstry, J. W. Belliveau, J. B. Moore, et al. J. Magn. Reson. Imaging 1(ab), 191 ( 1991 ).

43. K. K. Kwong, R. C. MCKinstry, D. CHIEn, AND B. R. Rosen, J. Magn. Reson. Imaging 1 (ab), 201 (1991).

44. J. J. NEIL, L. A. SCHERRER, AND J. J. H. ACKERMAN, "Book of Abstracts: Society of Magnetic Resonance in Medicine, 1991," p. 996. 